\title{
Streamer propagation in air
}

\section{R Morrow and $\mathbf{J} \mathbf{J}$ Lowke}

CSIRO Division of Applied Physics, Sydney, Australia 2070

Received 20 May 1996

\begin{abstract}
A theory is presented for the development of the first streamer when a positive voltage is abruptly applied to a point in air at atmospheric pressure. The continuity equations for electrons, positive ions and negative ions, including the effects of ionization, attachment, recombination, electron diffusion, and photoionization, are solved simultaneously with Poisson's equation. With an applied voltage of $20 \mathrm{kV}$ across a $50 \mathrm{~mm}$ gap, the streamer does not reach the cathode. An intense electric field front propagates away from the point into the gap to a distance of $35 \mathrm{~mm}$ in $200 \mathrm{~ns}$. During the next $7.5 \mu \mathrm{s}$ the streamer only moves a further $2 \mathrm{~mm}$ into the gap, and the electric field at the head of the streamer collapses. Finally, only positive space charge remains which moves away from the point, allowing the field near the point to recover after $\sim 10 \mu \mathrm{s}$; free electrons can thus give rise to a secondary discharge near the anode. The electric field distribution is shown to be quite different from that found previously for $\mathrm{SF}_{6}$ in that the electric field in the column of the streamer is generally only a fraction of the critical field for which ionization equals attachment. Streamers for a given applied voltage have a far greater range in air than in $\mathrm{SF}_{6}$. The results presented for air also apply to flue gas mixtures, since the important material properties of both gases are very similar.
\end{abstract}

\section{Introduction}

Recently there has been interest $[1,2]$ in the use of positivepulsed coronas for chemical processing, such as in the production of ozone from air or the reduction of the gaseous pollutants $\mathrm{SO}_{2}, \mathrm{NO}$, and $\mathrm{NO}_{2}$ (all of which cause acid rain) in flue gases. One method is to apply sub-microsecond positive-voltage pulses to the wires of an electrostatic precipitator. The authors have carried out experimental and theoretical studies of the effects of such voltage pulses applied to model precipitators [3-5].

Any detailed analysis of such plasma chemical processing involves a study of reactions of electrons and their products. Electron energies and number densities are dependent on the variation of the electric field in time. The present paper aims to predict the time and spatial dependence of the electric field and electron number density for a typical positive corona streamer pulse.

When a positive voltage is applied to a wire in air, the first corona phenomenon observed is the onset of a streamer from the wire, followed rapidly by the formation of the 'Hermstein glow' [6,7], an apparently continuous glow corona uniformly covering the wire, but not extending as far into the gap as onset streamers. However, when the positive voltage is raised in less than a microsecond the onset streamer can grow into a streamer which can propagate all the way across the gap. The same phenomena occur in flue gas mixtures. It is pointed out in section 3 that the electrical properties of air, important for describing streamer propagation, are very similar to those of flue gases; thus the streamer properties are expected to be very similar.
Calculations are presented in this paper for a case in which the streamer does not quite reach the anode. All the phases of streamer propagation are examined, i.e. an initial avalanche phase, a fast streamer propagation phase, a slow streamer propagation phase, a streamer termination phase, and the decay of the streamer channel. Results are obtained for the spatio-temporal distributions of electrons, positive ions, negative ions, the net charge, and the space charge distorted electric field. From the electric field distributions the most likely conditions for chemical reactions to be initiated can be deduced.

The calculations are performed by solving the coupled continuity equations for the development of electron and positive- and negative-ion densities coupled with Poisson's equation. In order to allow calculations of streamer propagation over long gaps $(\sim 50 \mathrm{~mm})$, with highly nonuniform electric fields, and for long time periods $(\sim 10 \mu \mathrm{s})$, one-dimensional forms of the continuity equations have been used. The use of one-dimensional forms of the continuity equations to describe streamer propagation, with a fixed channel radius, has been very successful [8-14], and has been used most recently by Aleksandrov and Bazelyan [15] to describe long-streamer propagation in air. The extra computation required for two-dimensional calculations has meant that, to date, the two-dimensional simulation of streamer propagation $[16,17]$ has been limited to short gaps $(\sim 5 \mathrm{~mm})$, and short time intervals $(\sim 7 \mathrm{~ns})$. From the twodimensional calculations, we can deduce that the streamer channel elongates as determined by the axial component of the continuity equation, once the radial structure is defined, and the radius is relatively fixed. 
The use of one-dimensional continuity equations allows a straightforward use of the photoionization data, rather than the use of a background pre-ionization electron density to provide seed electrons ahead of the streamer, as used in two-dimensional simulations [16,17], and in the long gap calculation of Aleksandrov and Bazelyan [15].

The effects of ionization, attachment, recombination and photoionization are included, as well as electron, positive-ion, and negative-ion drift, and electron diffusion. All these effects have a significant influence on the development of the discharge over the long timescales considered in this paper $(\sim 10 \mu \mathrm{s})$. Thus, it is inappropriate to make comparisons of the present results with the simple streamer models of D'yakonov and Kachorovskii [18] and Evlakhov et al [19], in which only ionization is considered in an initially uniform electric field, and drift motion is neglected.

\section{Theory}

It is known from observation that streamers occupy a narrow cylindrical channel between the anode and the cathode $[7,20]$. The electric field must be computed in two dimensions by solving Poisson's equation. The electron, positive-ion and negative-ion continuity equations including ionization, attachment, recombination and photoionization are solved simultaneously with Poisson's equation, but in one dimension as discussed above, to give electron and ion density distributions and the electric field. The solutions of the convective flow of particles are obtained using a flux-corrected-transport algorithm [21].

The coupled continuity equations for electrons, positive ions, and negative ions are:

$$
\begin{aligned}
\frac{\partial N_{e}}{\partial t} & =S+N_{e} \alpha\left|W_{e}\right|-N_{e} \eta\left|W_{e}\right|-N_{e} N_{p} \beta \\
& -\frac{\partial\left(N_{e} W_{e}\right)}{\partial x}+\frac{\partial}{\partial x}\left(D \frac{\partial N_{e}}{\partial x}\right) \\
\frac{\partial N_{p}}{\partial t} & =S+N_{e} \alpha\left|W_{e}\right|-N_{e} N_{p} \beta-N_{n} N_{p} \beta-\frac{\partial\left(N_{p} W_{p}\right)}{\partial x} \\
\frac{\partial N_{n}}{\partial t} & =N_{e} \eta\left|W_{e}\right|-N_{n} N_{p} \beta-\frac{\partial\left(N_{n} W_{n}\right)}{\partial x}
\end{aligned}
$$

where $t$ is the time; $x$ the distance from the anode; $N_{e}, N_{p}$ and $N_{n}$ the electron, positive-ion and negativeion densities, respectively; and $W_{e}, W_{p}$, and $W_{n}$ the electron, positive-ion and negative-ion drift velocities respectively. The symbols $\alpha, \eta, \beta$, and $D$ denote the ionization, attachment, recombination and electron diffusion coefficients respectively, and $S$ is the source term due to photoionization. Poisson's equation is:

$$
\nabla^{2} \phi=-\frac{e}{\epsilon}\left(N_{p}-N_{e}-N_{n}\right)
$$

where $\epsilon$ is the dielectric constant, $e$ the electron charge and $\phi$ the electric potential. The electric field, $E$, is computed using

$$
E=-\nabla \phi
$$

It is assumed that the transport properties of the gas (such as $\alpha$ and $W_{e}$ ) are determined by $E / N$, where $E$ is the local electric field and $N$ is the neutral gas number density. Detailed discussions of this assumption have been presented elsewhere [11,22].

Transport data for the material functions of air and flue gas mixtures come from previous calculations [4]. Little difference is found between the material functions of air and flue gas (i.e. $\alpha, \eta, W_{e}, D$, etc); thus the results of the calculations apply equally well to air or flue gas. The actual expressions fitted to the material function data, and used for these calculations, are tabulated in the appendix. The photoionization data of Penney and Hummert [23] for air are used, as well as their method of calculating photoionization densities [23, equation 2].

The continuity equation for electrons (equation (1)) is second order and therefore requires two boundary conditions: at the anode, $x=0, N_{e}=0$; and at the cathode, $x=6.5 \mathrm{~cm}, N_{e}=0$, since no secondary emission from the cathode is included. It is considered that processes at the cathode do not effect the discharge. The continuity equations for positive and negative ions (equations (2) and (3)) are both first order and thus require only one boundary condition each: at the cathode $N_{n}=0$; and at the anode $N_{p}=0$. At absorbing boundaries the heavy particle densities are finite and determined by the flux from the body of the discharge as discussed by Morrow [11].

The current, $I$, in the external circuit, due to the motion of electrons and ions between the electrodes, is calculated using Sato's equation [24], which is modified to include negative ions and electron diffusion:

$I=\frac{A e}{V_{a}} \int_{0}^{d}\left(N_{p} W_{p}-N_{n} W_{n}-N_{e} W_{e}+D \frac{\partial\left(N_{e}\right)}{\partial x}\right) E_{L} \mathrm{~d} x$.

Here $V_{a}$ is the applied voltage, $A$ the cross sectional area of the discharge channel and $E_{L}$ the Laplacian electric field.

It is assumed that $\psi$, the light intensity emitted by the discharge at any position, is proportional to the ionization activity, and may be written as

$$
\psi(x, t)=N_{e}(x, t) \alpha(x, t)|W e(x, t)| .
$$

This description has been used successfully to describe the output light intensity for a negative point-plane gap [11].

The numerical methods used to solve the continuity equations have been described in detail elsewhere $[21,22,25]$; it is only necessary here to point out that it is crucial for such long calculations, with $\sim 10^{7}$ time steps, to use a very accurate numerical algorithm, such as the third-order flux-corrected-transport algorithm used in this paper. However, it has been found that serious errors arise if the disc method [26] is used to compute the electric field for the case of air, with streamers crossing the entire gap. A successive over relaxation (SOR) method has been used instead in order to obtain the two-dimensional axisymmetric solution of the electric potential $\phi$ from Poisson's equation (4).

For fast-moving transient phenomena, such as streamer fronts, a moving computational mesh is usually required $[13,14]$. However, the continuous movement of the mesh is inappropriate for this calculation. Firstly, many geometric parameters would have to be recalculated at every time step, 


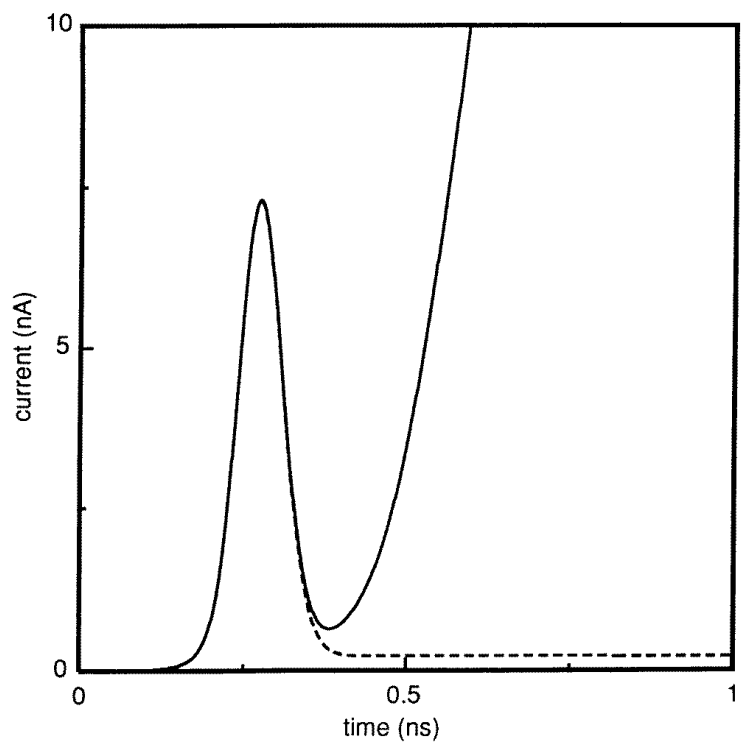

Figure 1. External circuit current for the first $1 \mathrm{~ns}$ after the release of seed electrons showing the pulse of current due to the first avalanche and subsequent current growth $(-)$, and the same calculation with photoionization set to zero $(---)$.

making the computation inefficient. Secondly, the electrons move in the opposite direction to that of the streamer front, increasing the effective value of $W_{e}$ relative to the mesh, and reducing the value of the time increment required for numerical stability.

The strategy adopted to follow the streamer front has been to use a fine uniform mesh, across which the streamer head propagates, and an expanding mesh away from this region towards the electrodes. When the streamer reaches the end of the fine mesh region, the mesh is re-zoned so that the streamer head is again at the start of the fine mesh region. No interpolation is required for the streamer head region since the fine mesh is uniform; linear interpolation is used elsewhere.

At the anode a fine mesh is also used to resolve the anode fall region. The mesh expands smoothly away from the anode, and then contracts towards the streamer head region. Similarly, the mesh expands away from the streamer head region towards the cathode, but with no fine mesh region at the cathode. The smooth changes and expansion of the mesh are achieved using exponential functions.

The total number of axial mesh points used to represent the $5 \mathrm{~cm}$ gap between the cathode and the anode is 400 . Relatively few mesh points serve to resolve the region between the streamer and the cathode. The mesh size at the streamer head is $1 \mu \mathrm{m}$, and other mesh sizes are similar to those discussed by Morrow [13,14]. The continuity equations are solved on this mesh of 400 points.

Poisson's equation is solved on a two-dimensional mesh, so radial dimensions have to be defined, and further axial dimensions defined so that the shape of the anode is included. The radial mesh has a minimum mesh size of $10 \mu \mathrm{m}$ at the centre, expanding exponentially with 50 mesh

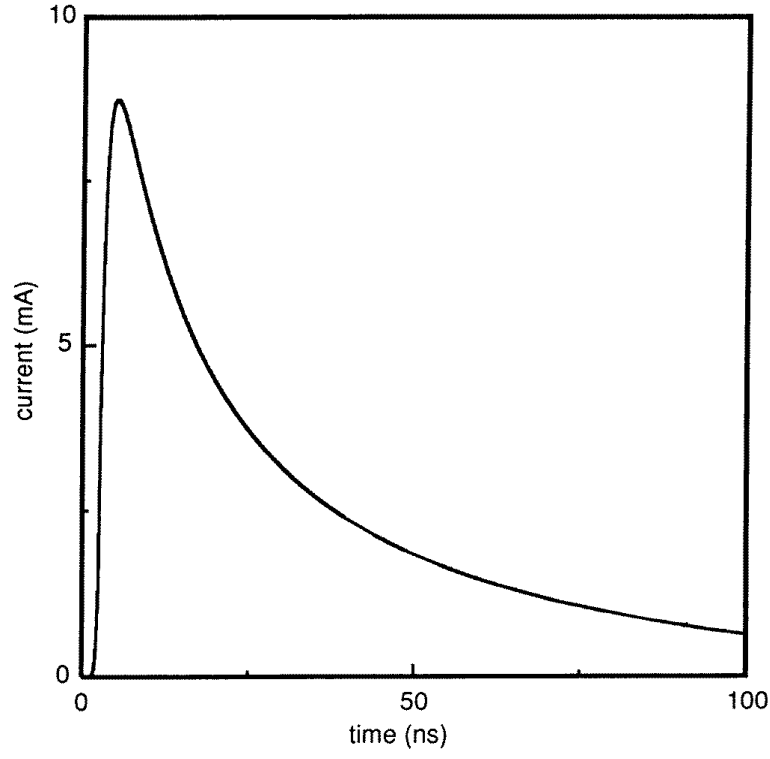

Figure 2. Total external circuit current, due to electron motion, for the first $100 \mathrm{~ns}$ of the discharge development.

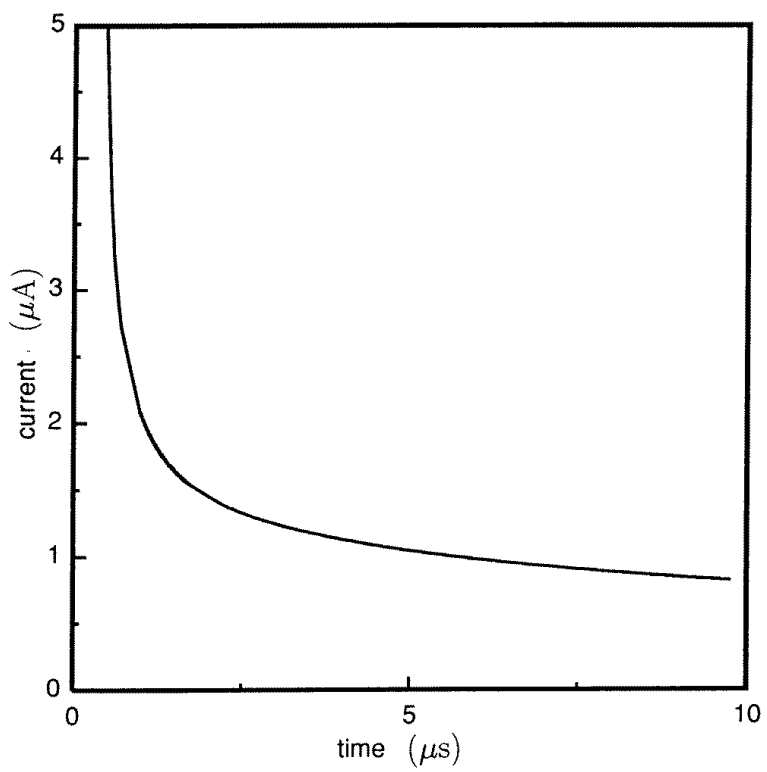

Figure 3. Total external circuit current over $10 \mu \mathrm{s}$, showing the transition from the high current, dominated by the electron contribution, to a relatively constant $\sim 1 \mu \mathrm{A}$ current due to the remnant positive ions in the streamer channel.

points out to a radius of $2 \mathrm{~cm}$. The shape of the anode is included accurately by defining a new axial mesh point for each radial mesh point such that the position defined by the axial and radial coordinates lies on the anode surface In this way $\sim 6.5 \mathrm{~cm}$ of the anode is included using an extra 40 mesh points (see figure 4, 5 and 6 later). Thus Poisson's equation is solved on a mesh of 440 axial points and 50 radial points in the region $0 \leq R \leq 20 \mathrm{~mm}$, and $0 \leq Z \leq 114 \mathrm{~mm}$, of figure 4 .

The boundary conditions used to solve Poisson's equation are that the potential on the anode surface is fixed 


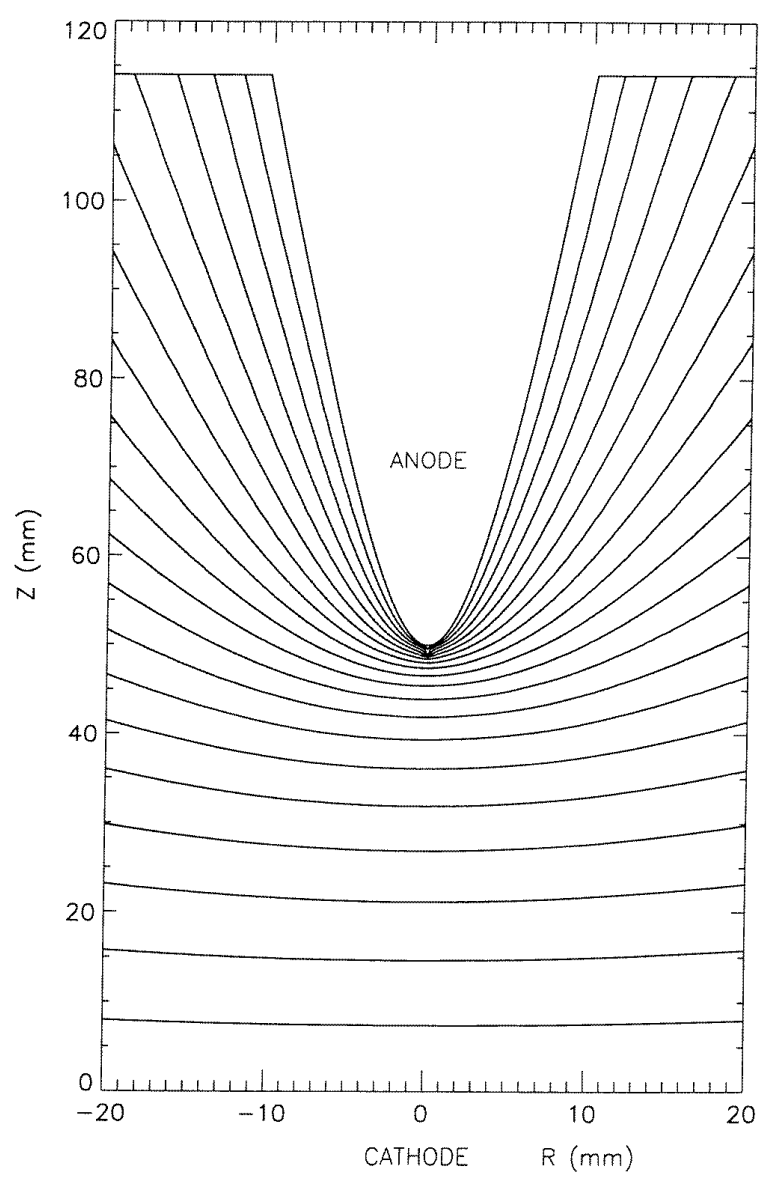

Figure 4. Electrical potential distribution after $3.3 \mathrm{~ns}$, showing the initial streamer formation, and the largely undisturbed initial potential distribution, with contours of equipotential differing by intervals of $1000 \mathrm{~V}$. The figure also shows the position and shape of the anode which is $50 \mathrm{~mm}$ from the cathode (at $Z=0 \mathrm{~mm}$ ). The anode is at $20 \mathrm{kV}$ and the cathode is at earth potential.

at $20 \mathrm{kV}$, and at the cathode surface the potential is set to zero. At the centre line ( $R=0$ in figure 4$)$ a symmetry boundary condition is used, $\partial \phi / \partial r=0$. This leaves the open boundaries to be defined at $10 \leq R \leq 20 \mathrm{~mm}$, $Z=114 \mathrm{~mm}$, and $R=20 \mathrm{~mm}, 0 \leq Z \leq 114 \mathrm{~mm}$, of figure 4 .

In order to provide accurate potential values at the open boundaries, and an exact solution of Laplace's equation for testing the SOR method of solution, a hyperboloid anodeplane cathode geometry is used for which an exact solution exists [27]. The free boundaries are placed well away from the space charge region so that any space charge effects can be neglected, and the exact solution to Laplace's equation is used to define the potential at the boundaries.

The exact solution is defined, following Erying et al [27], for positive potentials by the equation

$$
\phi(R, Z)=\left[-b+\left(b^{2}-4 a c\right)^{1 / 2} / 2 a\right]^{1 / 2}
$$

where $\phi$ is the potential at the point $(R, Z)$

$$
a=-Z_{0}^{2}\left(Z_{1}^{2}+R_{1}^{2}-Z_{0}^{2}\right) /\left(Z_{1}^{2}-Z_{0}^{2}\right)
$$

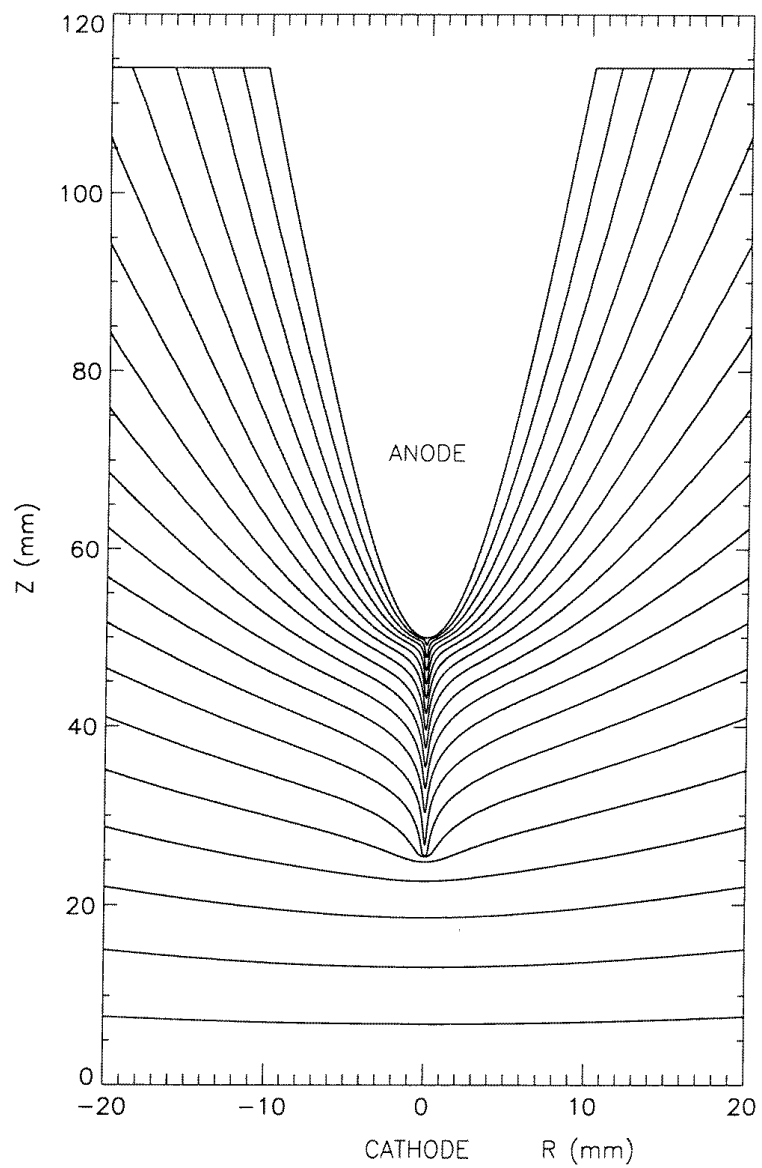

Figure 5. Potential distribution, with $1000 \mathrm{~V}$ equipotentials, after $54 \mathrm{~ns}$, showing the streamer $\sim 23 \mathrm{~mm}$ into the gap. Note the concentration of equipotential surfaces at the streamer tip, indicating a high $E$.

$$
b=Z^{2}+R^{2}+Z_{0}^{2}\left(Z_{1}^{2}+R_{1}^{2}-Z_{0}^{2}\right) /\left(Z_{1}^{2}-Z_{0}^{2}\right)
$$

and

$$
c=-Z^{2} \text {. }
$$

The electrode separation is $Z_{0}=50 \mathrm{~mm}$, and the anode shape is defined by $Z_{1}=51 \mathrm{~mm}$ and $R_{1}=1 \mathrm{~mm}$ specifying a point with $\sim 1 \mathrm{~mm}$ radius of curvature at the tip.

The central charge density is distributed radially using

$$
\rho(R, Z)=\rho_{a}(Z) \exp \left(-100 R^{2}\right)
$$

where $\rho_{a}(Z)$ is the axial charge distribution and $R$ is in millimetres. It is only necessary to set the radial charge out to the twelfth radial position $(\sim 300 \mu \mathrm{m})$, and the charge is set to zero beyond this point. In previous studies that used the disc method of solving Poisson's equation, the central charge was effectively used as the density over the the diameter of the channel $(100 \mu \mathrm{m})$, and the charge was set to zero beyond this diameter [12-14].

The numerical attachment instability [13] found during calculations in $\mathrm{SF}_{6}$ when $\delta x$, the mesh size, is greater than the channel diameter, $d$, has not been found in these calculations (even though $\delta x>d$ was used). The calculations are stable because attachment in air is much lower than that in $\mathrm{SF}_{6}$. 




Figure 6. Potential distribution, with $1000 \mathrm{~V}$ equipotentials, after $10 \mu \mathrm{s}$, when only positive ions remain; these ions move steadily away from the anode. Note that the equipotential surfaces have relaxed at the streamer head, indicating the collapse of the electric field in this region, while the equipotential surfaces have concentrated again near the anode in the gap left by the movement of positive ions in the streamer channel.

The calculations required the very powerful computing resources of a Cray-YMP computer for $\sim 60 \mathrm{~h}$. The total number of time steps was $\sim 10^{7}$. The computing time was dominated by the time required for the solution of Poisson's equation using the SOR method, which takes up to 50 iterations at each time step.

\section{Results}

Results are presented for the development of streamers from the positive point of a point-plane gap in air at atmospheric pressure. The point is a hyperboloid with a $\sim 1 \mathrm{~mm}$ radius of curvature at the tip. The gap spacing is $50 \mathrm{~mm}$, with a voltage of $20 \mathrm{kV}$ applied instantaneously at the start of the calculation.

First, details of the initial avalanche are shown, and the role of photoionization in streamer development outlined. Then the macroscopically observable results, such as the external circuit current, are discussed. The propagation of the streamer is introduced by outlining the two-dimensional

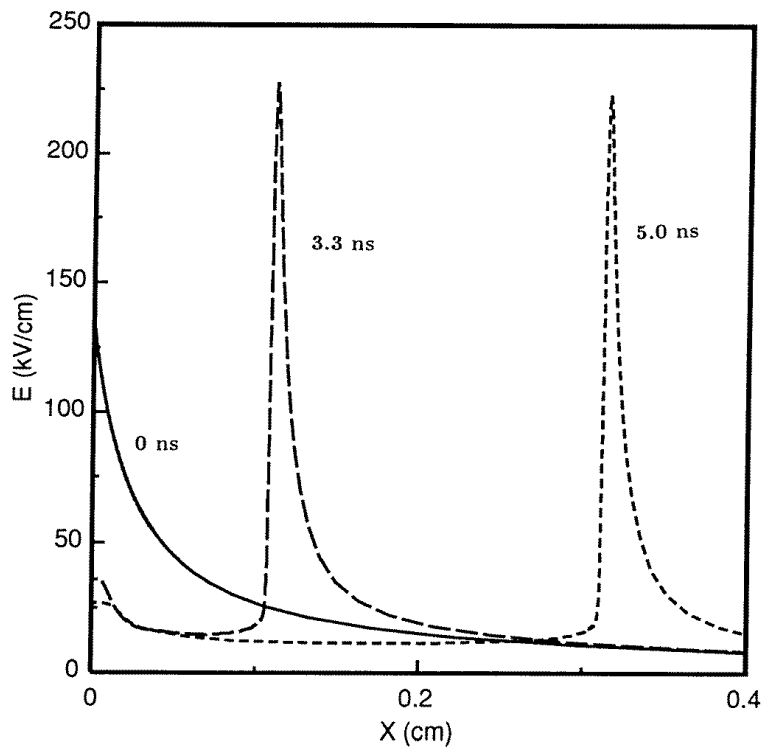

Figure 7. Electric field distributions along the axis of the discharge near the anode, at times indicated in nanoseconds.

distortion of the potential distribution due to the streamer space charge as the streamer moves away from the anode. The streamer development is then analysed in terms of successive distinct phases: initial streamer formation, rapid streamer propagation, slow streamer propagation, streamer termination, and finally, decay of the streamer channel. The detailed structure of the streamer front, while it is rapidly propagating, is analysed and contrasted with that found for $\mathrm{SF}_{6}$.

\subsection{The avalanche phase and the role of photoionization}

The calculation is initiated by $\sim 100$ electron-ion pairs released $0.1 \mathrm{~mm}$ from the anode at $t=0$. These electrons move rapidly into the high-field region near the anode tip, ionizing the gas and producing an increase in the numbers of electrons, forming the initial avalanche. The rise in electron numbers, and their motion in the high electric field near the anode, causes a rise in the external circuit current to a maximum of $7.5 \mathrm{nA}$, shown in figure 1 at $t=0.25 \mathrm{~ns}$. When electrons of the initial avalanche reach the anode they are absorbed, and the current falls. However, photoelectrons (due to photoionization) are distributed along the channel behind the avalanche as it proceeds towards the anode. These electrons multiply as they move into the high-field region near the anode, and the current rises again. For the initial conditions of the present calculation it takes $0.3 \mathrm{~ns}$ for the initial avalanche to reach its maximum current, and it takes a further $0.3 \mathrm{~ns}$ for the photoelectrons to supply a similar current. Because the photoelectrons are distributed along the channel no further current pulses are produced, and the current grows exponentially due to the formation of a selfsustained discharge. 




Figure 8. Electric field distributions computed from the potential distribution along the axis of the discharge using equation (5), at various times in nanoseconds, after the release of the initial electrons. The anode tip is at $X=0$ and the cathode at $X=5 \mathrm{~cm}$.

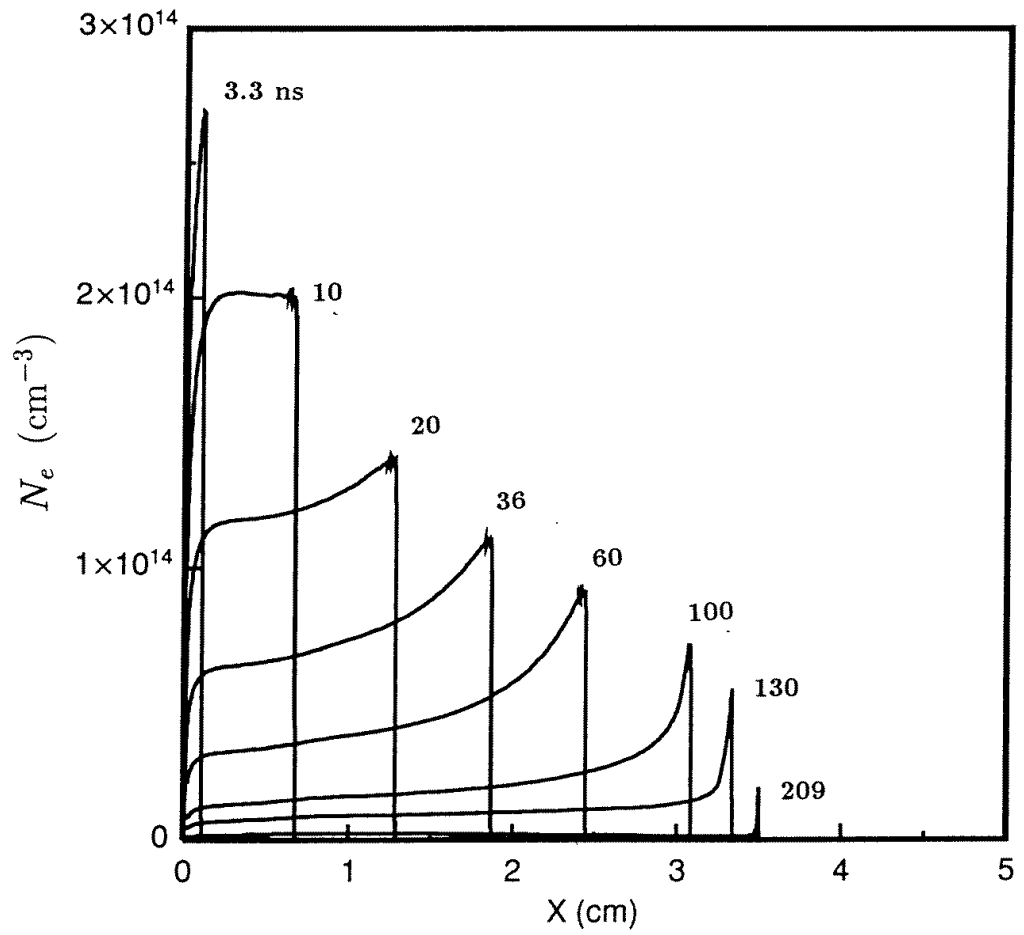

Figure 9. Electron density distributions computed at various times in nanoseconds.

The role of photoionization can be elucidated by setting it to zero. In figure 1 the results for zero photoionization are shown as a broken curve which overlaps the original curve completely for the first $\sim 0.35 \mathrm{~ns}$, indicating that the initial avalanche phase is identical in both cases. However, after $0.35 \mathrm{~ns}$ all the original electrons are absorbed into 


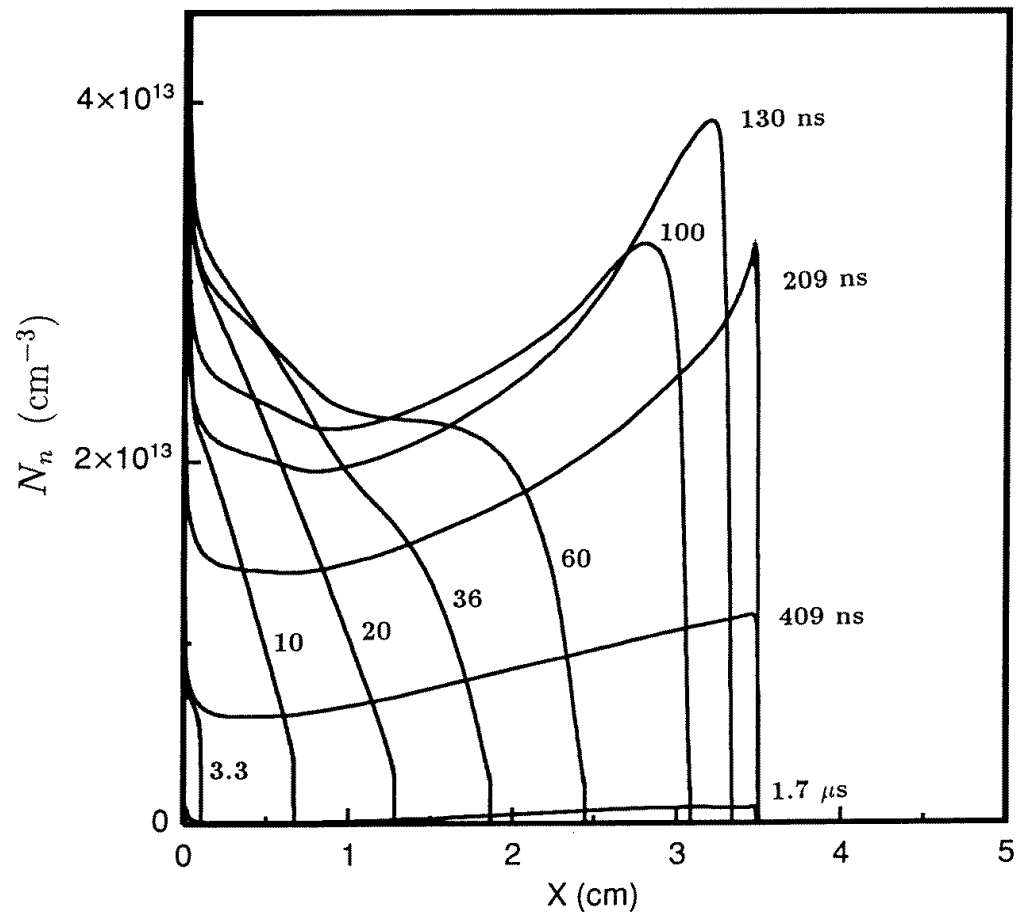

Figure 10. Negative-ion density distributions computed at various times in nanoseconds.

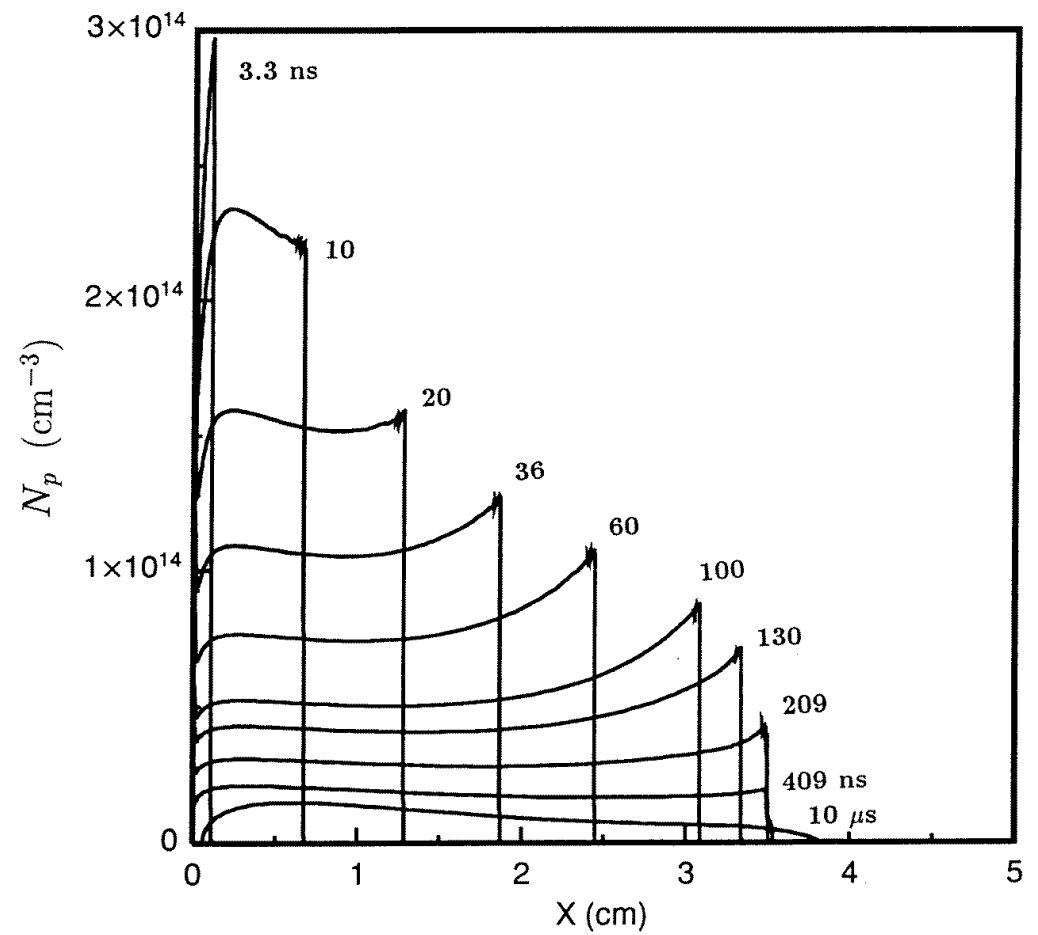

Figure 11. Positive-ion density distributions computed at various times in nanoseconds.

the anode, and there are no photoelectrons to sustain the discharge. After $0.35 \mathrm{~ns}$ the current is constant at $200 \mathrm{pA}$, being due to positive-ion and negative ion motion. Extreme care has been taken in using numerical methods which do not introduce non-physical effects, such as numerical diffusion, or spurious numerical oscillations $[21,22,25]$. The most important dynamic effects have been included in the calculations. Thus, these results demonstrate that 


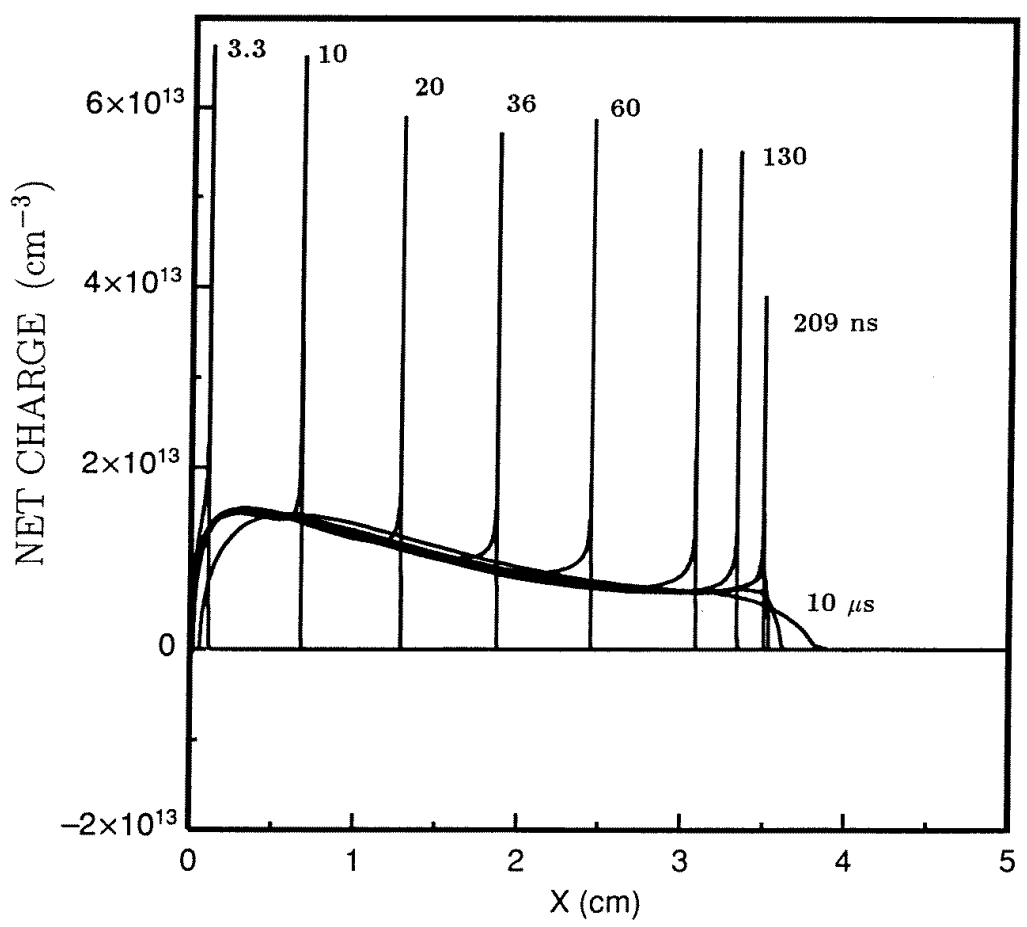

Figure 12. Net charge density distributions computed at various times in nanoseconds.

photoionization is essential for the formation of a selfsustained discharge at the anode.

\subsection{External circuit current}

The growth of the initial avalanche (discussed above) takes $\sim 2$ ns before the development of a significant current (figure 2). The external circuit current then rises steadily as shown in figure 2, to a maximum of $\sim 9 \mathrm{~mA}$, at $t=5 \mathrm{~ns}$, and then falls steadily, by an order of magnitude, during the next $100 \mathrm{~ns}$. The current continues to flow for the time span of the calculation $(\sim 10 \mu \mathrm{s})$ as shown in figure 3 . After $2 \mu \mathrm{s}$ the current falls from a peak of $9 \mathrm{~mA}$, due to electron motion, to $\sim 1 \mu \mathrm{A}$, due solely to positive-ion motion.

\subsection{Distortion of the electrical potential due to the streamer}

Before making a detailed analysis of the streamer structure it is informative to portray the progress of the streamer across the gap by showing, through a series of figures, how the streamer distorts the electrical potential distribution as it moves.

The potential distribution after $3.3 \mathrm{~ns}$ (figure 4) shows that the streamer front has propagated $\sim 1 \mathrm{~mm}$ into the gap at the tip of the point electrode, with the rest of the distribution left in its initial configuration. After $54 \mathrm{~ns}$ the streamer has propagated $23 \mathrm{~mm}$ into the gap (figure 5), with a concentration of potential contours at the streamer head, indicating a high electric field. The potential contours along the streamer channel are distorted by the presence of positive space charge.
The streamer stops after $\sim 200 \mathrm{~ns}$, having travelled to its full extent of $\sim 35 \mathrm{~mm}$, and the streamer channel starts to decay (see figure 14 later). After $10 \mu \mathrm{s}$ the equipotentials at the head of the streamer are no longer concentrated (figure 6), indicating a reduced electric field, and the equipotentials again concentrate at the tip of the anode indicating a rising electric field in this region.

\subsection{Detailed analysis of the streamer structure}

In order to analyse the streamer formation and propagation in detail the electric field along the centre line of the discharge is computed from the gradient of the potential distribution (equation (5)) for various times during the development of the discharge (figures 7 and 8). For the same times at which curves are displayed in figure 8, distributions of electrons, negative ions, positive ions, and the net charge are given in figures 9 to 12 respectively. A detailed analysis of the streamer structure at $t=36 \mathrm{~ns}$ is shown in figure 13. Figure 14 shows the collapse of the field at the streamer head at later times, and figure 15 the concomitant recovery of the field at the anode. Figure 16 shows the movement of positive charge away from the anode at later times, which causes the rising field near the anode. Finally, figure 17 shows the estimated intensity of the light from the streamer calculated using equation (21).

3.4.1. Initial streamer formation, $0-6 \mathrm{~ns}$. Consider the details of the profiles of figures 7-12 for $t=3.3 \mathrm{~ns}$. The charge density has risen, and space charge effects have lowered the electric field near the anode forcing a 'spike' in the electric field to move into the gap as an ionizing 


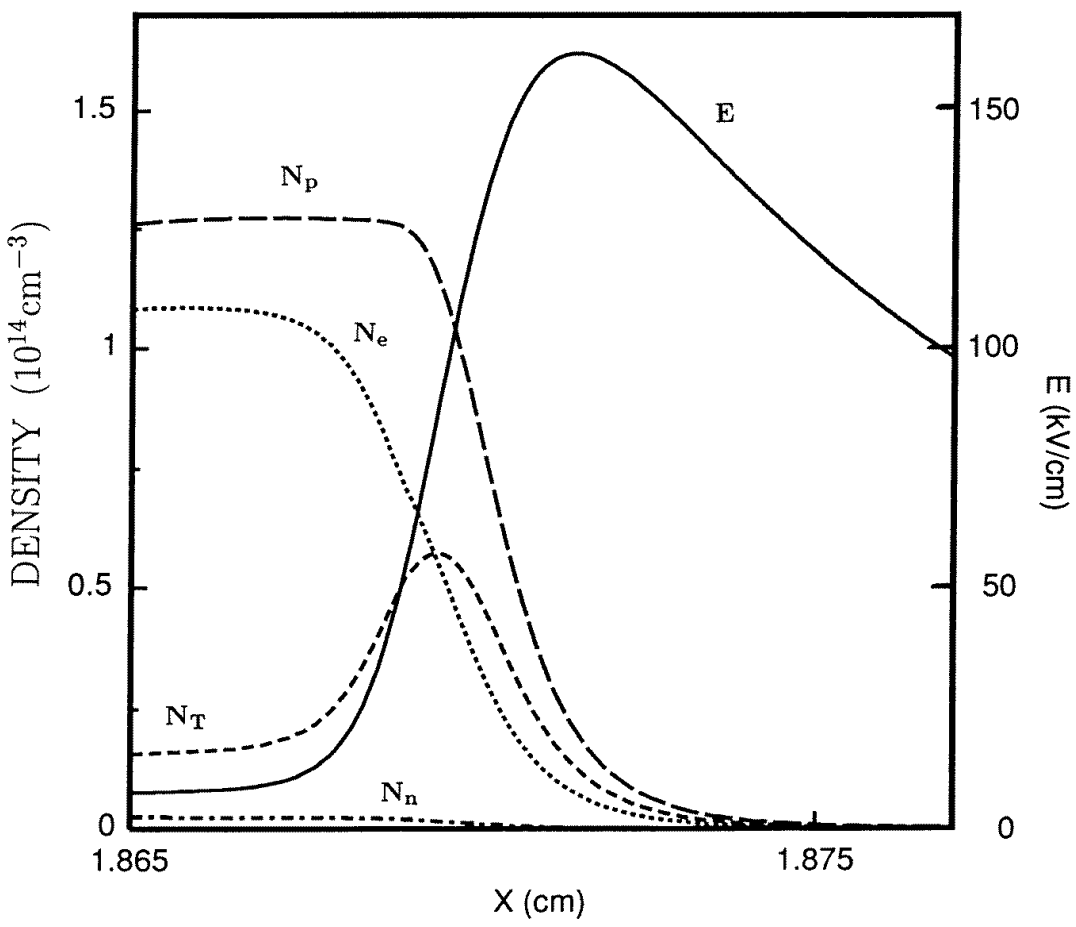

Figure 13. Structure of the streamer front at $t=36 \mathrm{~ns}$ : electric field, $E(-)$; electron density, $N_{e}(\cdots \cdots)$; negative ion density, $N_{n}(-\cdot-\cdot-)$; positive ion density, $N_{p}(---)$; net charge, $N_{T}(---)$.

wave (figures 7 and 8). The current is still rising, as seen in figure 2. However, the electric field in the streamer column is less than $30 \mathrm{kV} \mathrm{cm}^{-1}$ (figures 7 and 8), which is the field for which the ionization coefficient equals the attachment coefficient for air. The electron densities (figure 9), and positive-ion densities (figure 11) are $\sim 3 \times 10^{13} \mathrm{~cm}^{-3}$, while the negative-ion density is $<10^{13} \mathrm{~cm}^{-3}$ (figure 10). The net charge is positive (figure 12) due to loss of electrons to the anode, and is $\leq 10^{13} \mathrm{~cm}^{-3}$ except at the streamer head where there is a very sharp maximum of $7 \times 10^{13} \mathrm{~cm}^{-3}$.

3.4.2. Rapid streamer propagation, 6-40 ns. Consider the profiles at $t=36 \mathrm{~ns}$. The streamer velocity is very high; the streamer has travelled about $19 \mathrm{~mm}$, half its total transit distance, in $36 \mathrm{~ns}$, with an average velocity of $5.3 \times$ $10^{7} \mathrm{~cm} \mathrm{~s}^{-1}$ (figure 8). The magnitude of this high streamer velocity is in fair agreement with the experimental results of Allen and Ghaffar [28]. The electron density is $\sim 10^{14} \mathrm{~cm}^{-3}$ near the streamer head, but falls to $5 \times 10^{13} \mathrm{~cm}^{-3}$ along the channel; this fall is due to (1) negative ion formation from attachment, (2) recombination with positive ions, and (3) electron drift to the anode (figure 9). The negative ion density is correspondingly high near the anode $(\sim 3 \times$ $10^{13} \mathrm{~cm}^{-3}$ ), because the electrons have had sufficient time for negative ion formation, but decreases near the streamer head (figure 10). Recombination with positive ions also reduces the negative-ion densities, particularly when these densities are high. The electron number density is larger than the negative-ion density everywhere. The positive-ion density is $\sim 10^{14} \mathrm{~cm}^{-3}$ along the channel (figure 11), and has fallen from the higher values seen at $t=9.2$ and $22 \mathrm{~ns}$ due to recombination with electrons and negative ions.
The peak in the net charge at the streamer head is only slightly diminished from its initial value (figure 12). Behind the streamer a distribution of positive charge is laid out and remains relatively unchanged by the further progress of the streamer. The light output at $t=36 \mathrm{~ns}$ has fallen by a factor of six from that estimated at $t=3.3 \mathrm{~ns}$ (figure 17). Note that between $t=3.3 \mathrm{~ns}$ and $t=36 \mathrm{~ns}$ the external circuit current has been falling (figure 2), and that the electric field in the streamer channel is left relatively constant. The fall in the current is accounted for by the falling electron number densities during this time (figure 9). Because attachment is relatively slow in air, it is not necessary to create electrons in the streamer channel in order to maintain continuity of current. This contrasts with the situation in $\mathrm{SF}_{6}$ where $E \geq E^{*}$ must be maintained [12-14].

The detailed structure of a propagating streamer front at $t=36 \mathrm{~ns}$ is displayed in figure 13; a $100 \mu \mathrm{m}$ section of the streamer head shows variations in the particle number densities, the net charge, and the electric field through the ionizing wave front. The net positive space charge at the streamer head gives rise to an electric field extending far ahead of the streamer. In this high electric field region, electrons from photoionization produce a growth in the electron number density due to ionization. The electron number density, and corresponding positive ion density, do not reach a maximum until the electric field has fallen to less than half its maximum value. Note that the entire structure of figure 13 moves to the right. The negative-ion density increases so slowly with time that it plays no part in the dynamics of the streamer front. 




Figure 14. Collapse of the electric field at the streamer tip after streamer propagation has terminated. Times as indicated on the curves.

The streamer structure shown in figure 13 is very different from an analogous figure calculated for $\mathrm{SF}_{6}$ (see figure 7(a), Morrow [12]). For $\mathrm{SF}_{6}$ : (1) the negativeion density is always larger than the electron density; (2) positive- and negative-ion densities dominate the net charge in the streamer front; and (3) the distortion of the electric field is much less than that for air.

3.4.3. Slow streamer propagation, $40-130 \mathrm{~ns}$. Consider the profiles at $t=130 \mathrm{~ns}$. Between $t=36 \mathrm{~ns}$ and $t=130 \mathrm{~ns}$ the streamer slows down by a factor of four; it travels $15 \mathrm{~mm}$ in $94 \mathrm{~ns}$ with an average velocity of $1.6 \times 10^{7} \mathrm{~cm} \mathrm{~s}^{-1}$, and is near the end of its transit distance in the gap. The electric field amplitude in the streamer column stays relatively constant (figures 7 and 8), as does the net charge in the streamer head (figure 12).

Due to the slow progress of the streamer, the electron density (figure 9) is reduced by attachment and it is less than the negative-ion density (figure 10), except at the streamer head. The negative-ion distribution (figure 10) has changed character due to recombination with positive ions; the density near the anode has decreased, and the maximum is now near the streamer head (figure 9). The positive-ion density and the net positive charge both remain relatively constant (figure 12).

At an early time in the discharge, such as at $t=100 \mathrm{~ns}$ in figure 16, the movement of positive ions away from the anode and the net movement of electrons and negative ions towards the anode leads to a net negative space charge near the anode, and a corresponding sharp rise in the electric field (figure 15).
The light output is predicted to fall rapidly by two orders of magnitude from that at $t=3.3 \mathrm{~ns}$ (figure 17).

3.4.4. Streamer termination, 130-210 ns. Consider the profiles at $t=209 \mathrm{~ns}$. Between $t=130 \mathrm{~ns}$ and $t=209 \mathrm{~ns}$, the streamer moves only $1.6 \mathrm{~mm}$, with an average velocity of $2 \times 10^{6} \mathrm{~cm} \mathrm{~s}^{-1}$; thus the streamer propagation velocity has fallen by a factor of ten (figure 8). There is a decrease in the electric field (figure 8), and also in the positive charge spike (figure 12) at the streamer head. The electron density (figure 9) is very low everywhere in the channel, except for a sharp spike at the streamer head. However, electrons contribute significantly to the external current for $\sim 1 \mu$ s (figure 3 ). The negative-ion density exceeds the electron density everywhere in the channel (figure 10), and is comparable with the positive-ion density distribution (figure 11) which is relatively flat. The positive net charge behind the streamer head (figure 12) is relatively constant. The light output falls by two orders of magnitude between $t=130 \mathrm{~ns}$ and $t=209 \mathrm{~ns}$, and becomes negligible after that time.

At $t=400 \mathrm{~ns}$ a net negative space charge remains at the anode (figure 16), and the electric field at the anode increases with time (figure 15).

3.4.5. Decay of the streamer channel, $210 \mathrm{~ns}-10 \mu \mathrm{s}$. After $t=209 \mathrm{~ns}$ the electron number density is negligible (figure 9), and the streamer has stopped propagating (figure 8). The negative-ion number density is significant at $t=406 \mathrm{~ns}$ (figure 10), but after $t=2 \mu \mathrm{s}$ is negligible due to recombination with positive ions. Thus, after $t=2 \mu \mathrm{s}$, 


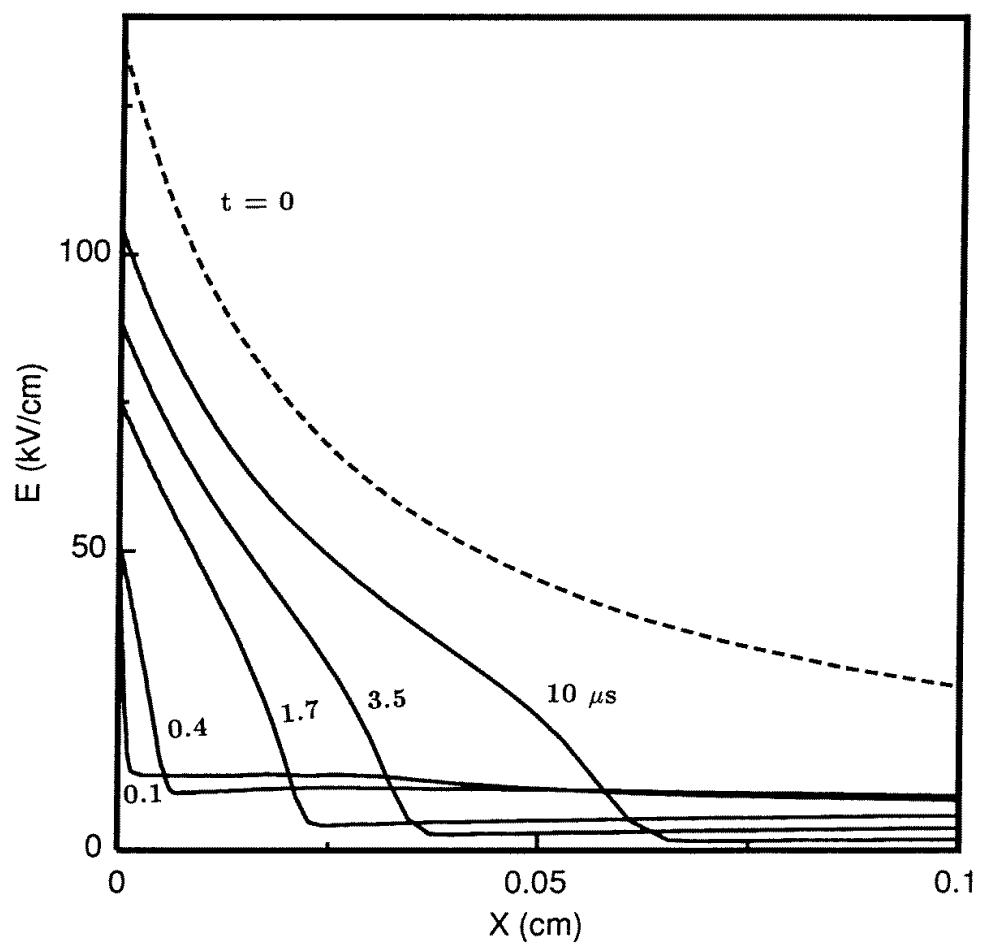

Figure 15. Development of the electric field at the anode as a function of time $(\mu \mathrm{s})$, showing the initial electric field, the rising electric field due to negative space charge, and the resurgence of the Laplacian electric field near the anode at late times due to the movement of positive space charge away from the anode.

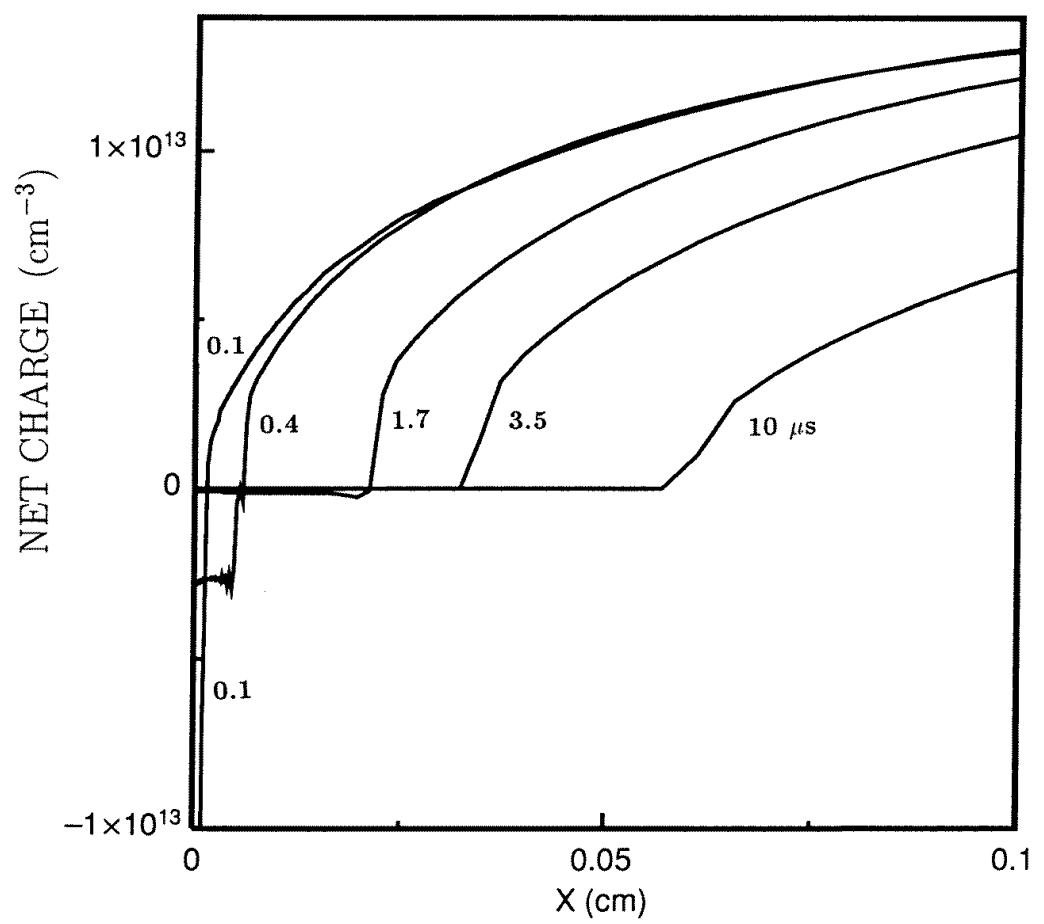

Figure 16. Details of the negative space charge near the anode at early times, and the movement of the remnant positive space charge away from the anode at later times.

the positive-ion distribution (figure 11), and the net charge distribution (figure 12) are identical. The small electric current of $\sim 1 \mu \mathrm{A}$, after $t=2 \mu$ s (figure 2), is therefore due only to the movement of positive ions. The small changes in the net charge distribution after $1 \mu \mathrm{s}$, shown in figure 12 , are due to the movement of the net positive 


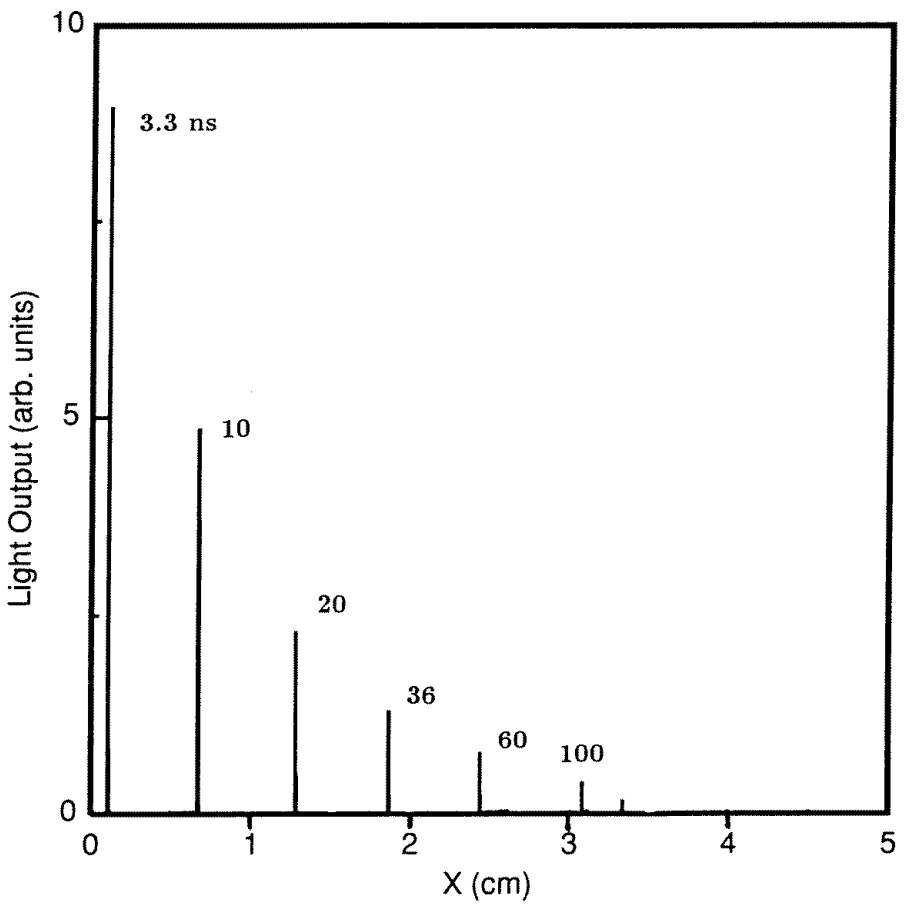

Figure 17. Light output $\psi$ at various times in nanoseconds, assumed to be proportional to the net ionization activity at a given time; $\psi=N_{e} \alpha\left|w_{e}\right|$.

charge away from the anode. This movement causes a slow dispersal of the electric field in the streamer head over a larger distance (figure 14). There is still a small net negative space charge at the anode at $t=1.7 \mu$ s (figure 16). However, after $10 \mu$ s only positive space charge remains and as this moves away from the anode (figure 16) the electric field approaches the magnitude and shape of the initial Lapacian field distribution (figure 15).

\section{Discussion}

The accuracy of the numerical methods that have been developed for the present calculations has enabled calculations of the development of discharges to be made for time periods up to $10 \mu \mathrm{s}$.

The calculation is initiated by 100 electron-ion pairs (representing ionization of the gas due to a cosmic ray, or radioactivity) released $0.1 \mathrm{~mm}$ from the electrode. In a real gas the number of electron-ion pairs and their position will vary statistically; this variation is not included in this calculation. If either the number of initial electrons or their position is changed in the calculation, then the time from initiation to, for example, the current maximum, varies. However, the maximum current, and the details of the streamer propagation do not change, as these properties are determined by space charge effects and are insensitive to the initial conditions.

The calculation is started with the full voltage already applied, corresponding to the case where the voltage reaches its maximum value before seed electrons are available. The case where the voltage rises in a finite time, with seed electrons available initially, has been considered in detail for $\mathrm{SF}_{6}$ [13], and similar considerations will apply for air.

The calculations include electron attachment coefficients suitable for air and flue gases. The effects of stronger attachment (due perhaps to higher water contents in exhaust gases) can be inferred by comparing the present results with those for $\mathrm{SF}_{6}[13,14]$.

The initial current pulse, typically found at the start of experimentally measured positive streamer current pulses (see for example [29]), is explained as the current due to an initial avalanche of electrons in the high-field region, triggered by a seed electron.

The main current pulse of amplitude $\sim 9 \mathrm{~mA}$, with a half width of $\sim 50 \mathrm{~ns}$, agrees qualitatively with pulses measured for positive corona in air. From the particle density results it is found that the relatively steady current of $\sim 1 \mu \mathrm{A}$ at late times, after the main pulse, is due to the motion of positive ions left in the gap.

In air at atmospheric pressure, the critical field $E^{*}$, at which the ionization coefficient equals the attachment coefficient, is $E^{*}=30 \mathrm{kV} \mathrm{cm}-1$; for $\mathrm{SF}_{6} E^{*}=$ $90 \mathrm{kV} \mathrm{cm}^{-1}$. In $\mathrm{SF}_{6}$, it was found that the electric field had to be maintained at $E \geq E^{*}$ in the streamer column in order to maintain conductivity while the streamer was propagating [12-14]: this is not the case for air. The lower attachment coefficient in air means that electrons are not lost rapidly if $E<E^{*}$. For example, in air, the attachment time constant, $\tau=1 /\left(\eta\left|W_{e}\right|\right)$, is $\sim 100 \mathrm{~ns}$ at $E=10 \mathrm{kV} \mathrm{cm}^{-1}$, and is similar to the propagation time of $100 \mathrm{~ns}$. On the other hand, for $\mathrm{SF}_{6}, \tau=0.06 \mathrm{~ns}$ at $E^{*}$ which is the electric field in the streamer channel [1214]. Thus attachment does not dominate the dynamics of streamer propagation in air as it does in $\mathrm{SF}_{6}$. 
The net charge density in the streamer head remains relatively constant as the streamer propagates, decreasing only slowly with time (figure 12). The electric field in the streamer head changes more significantly (figure 8), because it depends on the integrated charge in the streamer head, rather than on the peak charge. The extreme variation in ionization activity with electric field is shown in figure 17 for the light output, since the light output is proportional to the amount of ionization activity.

For applications where it is desirable to have a large electric field to promote specific chemical reactions, e.g. to reduce $\mathrm{NO}_{x}$ and $\mathrm{SO}_{x}$ in flue gases, the most important phase is probably the 'slow streamer propagation' phase ( $t=36 \mathrm{~ns}$ to $200 \mathrm{~ns}$ ). The electric field is relatively large, and remains at each location for a longer time. It is very likely that the early streamer propagation is not productive in generating free radicals because of the short duration $(\sim 100 \mathrm{~ns})$. Perhaps with a suitably shaped voltage waveform, streamers could be initiated and propagated at an ideal slow velocity. Note that the electric field in the column is generally very much less than the $30 \mathrm{kV} \mathrm{cm}^{-1}$ necessary to promote plasma chemical reactions [4].

The calculated results for light output agree qualitatively with the experimental observation of the streamer head propagating like a moving point of light [7, 28,30]. After $130 \mathrm{~ns}$ the calculated light output is difficult to detect photographically.

The movement of the remnant positive space charge away from the positive electrode can be related to the development of the 'corona wind'. The positive ions have a mass of the same order as that of the neutral particles and therefore transfer their momentum efficiently to the background gas.

When the positive charge moves away from the anode at later times, the field at the head of the avalanche collapses, while the electric field near the anode rises. This rising field near the anode may explain the observation of continuing luminosity near the anode in the streamer channel, from 10-100 $\mu \mathrm{s}$, after the passage of the streamer [31].

During a positive streamer discharge from a point, the electric field near the anode at first falls due to the shielding effect of the net positive charge of the discharge, but then the electric field tends to rise towards the anode for three different reasons.

(1) Very close to the anode the electron density decreases towards the anode until the boundary condition $N_{e}=0$ is satisfied at the anode surface, and the electric field rises in order to maintain current continuity, as outlined in detail for $\mathrm{SF}_{6}$ by Morrow [13].

(2) On a larger scale, a negative space charge layer at the anode leads to a negative gradient of the electric field (equation (5)), and hence a rising electric field towards the anode.

(3) At later times, when only positive space charge remains (moving away from the anode), the electric field rises at the anode as the electric field approaches the Laplacian distribution.

\section{Conclusions}

The calculations give detailed predictions of electron and ion densities and electric fields as functions of time and position. The time span extends from the initial streamer development, with a resolution of nanoseconds, to a total time for streamer development of $10 \mu \mathrm{s}$. Spatial resolution over the electrode separation of $5 \mathrm{~cm}$ has been obtained using mesh sizes of $1 \mu \mathrm{m}$ to resolve the features of the streamer head.

The results were obtained by solving one-dimensional particle continuity equations, and a two-dimensional solution of Poisson's equation, to give predictions of the dominant physical processes in positive corona. Crucial to the success of the model for such long calculations has been the use of very accurate flux-corrected-transport algorithms for particle transport, and an accurate two-dimensional solution of Poisson's equation.

The calculations elucidate a number of crucial features of positive streamer corona in air and flue gas.

(1) Photoionization is essential for the development of corona for a positive point-plane gap, and for the development and propagation of positive streamers. Photoionization enables seed electrons to be in the highfield region ahead of the maximum positive space charge at the streamer head.

(2) Attachment of electrons to form negative ions does not dominate the streamer propagation to the extent of requiring the critical field in the streamer channel because the typical attachment time of $\sim 100 \mathrm{~ns}$ is slow compared with typical propagation times. The electric field in the streamer channel behind the streamer head is predicted to be $\sim 5 \mathrm{kV} \mathrm{cm}{ }^{-1}$, even though the critical field, at which ionization equals attachment, is $\sim 30 \mathrm{kV} \mathrm{cm}^{-1}$. Because of the finite electric field in the plasma channel, streamer propagation eventually ceases when the entire applied voltage is required for the channel.

(3) Predicted streamer propagation velocities of $\sim 10^{8} \mathrm{~cm} \mathrm{~s}^{-1}$ are in fair agreement with experimental results.

(4) The streamer, for the conditions of the present calculations of $20 \mathrm{kV}$ across a $50 \mathrm{~mm}$ gap, is predicted to cease propagation after it has traversed $3.5 \mathrm{~cm}$ of the gap, in agreement with previously published experimental observations.

\section{Acknowledgments}

The authors would like to acknowledge the support of Mr C A J Paulson and Mr A J Prokopiuk during the course of this work, and Drs A J D Farmer, A B Murphy, G N Haddad and Mrs V M Bowers Morrow for help in editing the manuscript. This work was supported financially by the Commonwealth Department of Primary Industry and Energy through a Coal Research Development and Demonstration Grant. 


\section{Appendix}

The material functions for air may be represented by the following equations, where $E^{\prime}=|E|$ for economy of notation.

$$
\begin{aligned}
& \alpha / N=2.0 \times 10^{-16} \exp \left[\left(-7.248 \times 10^{-15} /\left(E^{\prime} / N\right)\right] \mathrm{cm}^{2}\right. \text { (A1) } \\
& E^{\prime} / N>1.5 \times 10^{-15} \mathrm{~V} \mathrm{~cm}^{2} \\
& =6.619 \times 10^{-17} \exp \left[-5.593 \times 10^{-15} /\left(E^{\prime} / N\right)\right] \mathrm{cm}^{2}(\mathrm{~A} 2) \\
& E^{\prime} / N \leq 1.5 \times 10^{-15} \mathrm{~V} \mathrm{~cm}^{2} \\
& \eta_{2} / N=8.889 \times 10^{-5}\left(E^{\prime} / N\right)+2.567 \times 10^{-19} \mathrm{~cm}^{2} \\
& E^{\prime} / N>1.05 \times 10^{-15} \mathrm{~V} \mathrm{~cm}{ }^{2} \\
& =6.089 \times 10^{-4}\left(E^{\prime} / N\right)-2.893 \times 10^{-19} \mathrm{~cm}^{2} \\
& E^{\prime} / N \leq 1.05 \times 10^{-15} \mathrm{~V} \mathrm{~cm}^{2} \\
& \eta_{3} / N^{2}=4.7778 \times 10^{-59}\left(E^{\prime} / N\right)^{-1.2749} \mathrm{~cm}^{5} \\
& \beta=2.0 \times 10^{-7} \mathrm{~cm}^{3} \mathrm{~s}^{-1} \\
& D=\left[0.3341 \times 10^{9}\left(E^{\prime} / N\right)^{0.54069}\right]\left|W_{e} / E\right| \mathrm{cm}^{2} \mathrm{~s}^{-1} \\
& W_{e}=-\left(E / E^{\prime}\right)\left[7.4 \times 10^{21}\left(E^{\prime} / N\right)+7.1 \times 10^{6}\right] \mathrm{cm} \mathrm{s}^{-1} \\
& E^{\prime} / N>2.0 \times 10^{-15} \mathrm{~V} \mathrm{~cm}^{2} \\
& =-\left(E / E^{\prime}\right)\left[1.03 \times 10^{22}\left(E^{\prime} / N\right)+1.3 \times 10^{6}\right] \mathrm{cm} \mathrm{s}^{-1} \\
& 10^{-16} \leq E^{\prime} / N \leq 2.0 \times 10^{-15} \mathrm{~V} \mathrm{~cm}^{2} \\
& =-\left(E / E^{\prime}\right)\left[7.2973 \times 10^{21}\left(E^{\prime} / N\right)\right. \\
& \left.+1.63 \times 10^{6}\right] \mathrm{cm} \mathrm{s}^{-1} \\
& 2.6 \times 10^{-17} \leq E^{\prime} / N \leq 10^{-16} \mathrm{~V} \mathrm{~cm}^{2} \\
& =-\left(E / E^{\prime}\right)\left[6.87 \times 10^{22}\left(E^{\prime} / N\right)+3.38 \times 10^{4}\right] \mathrm{cm} \mathrm{s}^{-1} \\
& E^{\prime} / N \leq 2.6 \times 10^{-17} \mathrm{~V} \mathrm{~cm}^{2} \\
& W_{n}=-2.7 \times E P_{0} / P \mathrm{~cm} \mathrm{~s}^{-1} \\
& E^{\prime} / N>5.0 \times 10^{-16} \mathrm{~V} \mathrm{~cm}^{2} \\
& =-1.86 \times E P_{0} / P \mathrm{~cm} \mathrm{~s}^{-1} \\
& E^{\prime} / N<5.0 \times 10^{-16} \mathrm{~V} \mathrm{~cm}^{2}
\end{aligned}
$$

Note $P_{0} / P$ is the ratio of atmospheric pressure to the gas pressure, $\eta_{2}$ is the two-body attachment coefficient and $\eta_{3}$ is the three-body attachment coefficient.

\section{References}

[1] Penetrante B M and Schultheis S E (ed) 1993 Non-Thermal Plasma Techniques for Pollution Control, NATO ASI Series Parts A and B (Berlin: Springer)

[2] Masuda S Non-Thermal Plasma Techniques for Pollution Control, NATO ASI Series ed B M Penetrante and S E Schultheis Part B (Berlin: Springer) p 131

[3] Morrow R, Lowke J J, Paulson C A J and Prokopuik A J 1994 Electrical Engingeering Congress (Sydney) (Barton, ACT: The Institute of Engineers) pp 241-6

[4] Lowke J J and Morrow R 1995 IEEE Plasma Sci. 23 $661-71$

[5] Lowke J J and Morrow R 1995 Aust. J. Phys. 48 403-9

[6] Cross J and Beattie J 1980 Can. Elec. Eng. J. 522

[7] Meek J M and Craggs J D (ed) 1978 Electrical Breakdown of Gases (New York: Wiley)

[8] Davies A J, Davies C J and Evans C J 1971 Proc. Inst. Electr. Eng. 118816

[9] Kline L E 1974 J. Appl. Phys. 452046

[10] Abbas I and Bayle P 1980 J. Phys. D: Appl. Phys. 131055

[11] Morrow R 1985 Phys. Rev. A 321799

[12] Morrow R 1987 Phys. Rev. A 351778

[13] Morrow R 1991 IEEE Trans. Plasma. Sci. 19 86-94

[14] Morrow R 1991 IEEE Trans. Elec. Insul. 26 398-404

[15] Aleksandrov N L and Bazelyan E M 1996 J. Phys. D: Appl. Phys. 29 740-52

[16] Dhali S K and Williams P F 1987 J. Appl. Phys. 62 4696-707

[17] Vitello P A, Penetrante B M and Bardsley J N 1994 Phys. Rev. E 49 5574-98

[18] D'yakonov M I and Kachorovskii V Y 1989 Sov. Phys.-JEPT 68 1070-4

[19] Evlakhov N V, Kachorovskii V Y and Chistyakov V M 1992 Sov. Phys.-JEPT 75 31-6

[20] Morrow R 1988 J. Appl. Phys. 63 5171-4

[21] Morrow R and Noye J 1991 Computational Techniques and Applications Conference ed J Noye, P Benjamin and L Colgan, pp 347-54

[22] Morrow R 1987 XVIII Int. Conf. on Phenomena in Ionised Gases (Swansea, Wales) Invited papers ed W T Williams (Bristol: Adam Hilger) p 268

[23] Penney G W and Hummert G T 1970 J. Appl. Phys. 41572

[24] Sato N 1980 J. Phys. D: Appl. Phys. 13 L3

[25] Morrow R and Steinle P 1989 Computational Techniques and Applications: CTAC-89 ed W L Hogarth and J Noye (Amsterdam: North-Holland) 327-32

[26] Davies A J and Evans C J 1967 Proc. Inst. Electr. Eng. 1141547

[27] Eyring C F, Mackeown S S and Millikan R A 1928 Phys. Rev. 31900

[28] Allen N L and Ghaffar A 1995 J. Phys. D: Appl. Phys. 28 $338-43$

[29] McAllister I W, Crichton G C and Bregnsbo E 1979 J. Appl. Phys. 50 6797-805

[30] Creyghton Y, Smeets B, van Veldhuizen E M and Rutgers W R 1992 Proc. Tenth Int. Conf. on Gas Discharges and their Applications (Swansea, Wales) ed W Terry Williams (Local Organizing Committee of GD92)

[31] Lamb D W 1992 Optical studies of positive coronas in air $P h D$ Thesis University of New England, Australia 